\title{
Detección de la descarga subterránea al mar en la costa bonaerense mediante el uso de ${ }^{222} \mathrm{Rn}$ y métodos geoeléctricos
}

\author{
Silvina Carretero(1), Santiago Perdomo(2,3), John Rapaglia(4), Carlos Albino Martínez ${ }^{(3)}$ y
} Eduardo Kruse ${ }^{(1)}$

(1) CONICET, CEIDE, Facultad de Ciencias Naturales y Museo, Universidad Nacional de La Plata (UNLP), $64 n^{\circ}$ 3, 1900 La Plata, Argentina. scarretero@fcnym.unlp.edu.ar; kruse@fcnym.unlp.edu.ar

(2) CONICET, CITNOBA, Universidad Nacional del Noroeste de la Provincia de Buenos Aires (UNNOBA), Monteagudo 2772. Pergamino. Buenos Aires.

(3) Facultad de Ciencias Astronómicas y Geofísicas, Paseo del Bosque s/n. La Plata. Buenos Aires. Argentina. sperdomo@carina.fcaglp.unlp.edu.ar; albinocarlos7@gmail.com

(4) Biology Department, Sacred Hear University, 5151 Park Ave, Fairfield, CT 06825, EE. UU. john.rapaglia@gmail.com

\author{
RESUMEN
}

La descarga de agua dulce subterránea en el mar (SGD: sigla en inglés) es un proceso hidrológico complejo que ocurre en la interfaz continente-océano y juega un papel importante en la dinámica costera. En la costa oriental de la provincia de Buenos Aires (Argentina) se planteó como objetivo detectar la descarga de agua dulce desde el acuífero arenoso hacia el Océano Atlántico. Existen diversas metodologías utilizadas para detectar la SGD. En este trabajo se ha aplicado la técnica del ${ }^{222} \mathrm{Rn}$ como trazador, tomografía de resistividad eléctrica (ERT) y mapas de flujo subterráneo. Se midió la actividad del ${ }^{222} \mathrm{Rn}$ en el agua subterránea en perforaciones, en la playa (zona intermareal y de surf) y en una transecta a $200 \mathrm{~m}$ de la línea de costa donde conjuntamente se aplicó el método geoeléctrico. Se midió la profundidad en los pozos de la red de monitoreo existente y se construyeron mapas isofreáticos. La actividad del ${ }^{222} \mathrm{Rn}$ en las perforaciones oscilan entre 16 y $173 \mathrm{dpm} / \mathrm{L}$, en la costa entre 28 y $48 \mathrm{dpm} / \mathrm{L}$, y a lo largo de la transecta entre 1,3 y $20,5 \mathrm{dpm} / \mathrm{L}$. La ERT muestra una capa de alta resistividad cercana a los 3-4 $\mathrm{m}$ de profundidad respecto del fondo marino, la cual indicaría la presencia de agua dulce. Los mapas de flujo muestran descarga hacia la llanura deprimida al oeste y hacia el mar al este. No existen antecedentes de aplicación conjunta de estas metodologías en el área, lo cual resulta en una contribución de interés para el conocimiento de la hidrodinámica costera.

Palabras clave: acuífero costero, Buenos Aires, descarga subterránea, radón, tomografía de resistividad eléctrica.

\section{Submarine groundwater discharge detection on the coast of Buenos Aires using ${ }^{222} \mathrm{Rn}$ and geoelectrical methods}

\begin{abstract}
Submarine groundwater discharge (SGD) is a complex hydrological process which occurs in the continentocean interface and plays an important role in coastal dynamics. The detection of groundwater discharge from the sandy freshwater aquifer towards the Atlantic Ocean was proposed on the western coast of Buenos Aires Province (Argentina). There are different methods used to detect SGD. In this study, ${ }^{222} R n$ as a tracer, electrical resistivity tomography (ERT) and flow maps were used as the methodology. ${ }^{222}$ Rn activity was measured in the wells, at the beach (tidal pools and surf zone) and along a transect $200 \mathrm{~m}$ from the coastline where geo-electrical method was also used. Groundwater depth was measured in the wells and groundwater contour maps were made. ${ }^{222} \mathrm{Rn}$ activity in the wells varies from 16 and $173 \mathrm{dpm} / \mathrm{L}$, at the beach the values are between 28 and $48 \mathrm{dpm} / \mathrm{L}$ and along the coastline they oscillate between 1.3 and $20.5 \mathrm{dpm} / \mathrm{L}$. The ERT shows a high resistivity layer close to a depth of 3-4 $\mathrm{m}$ from the sea floor, which would indicate the presence of freshwater. Groundwater contour maps show discharge toward the continental plain to the west and toward
\end{abstract}


Carretero, S. Detección de la descarga subterránea al mar en la costa bonaerense... Boletín Geológico y Minero, 132 (1-2): 157-166

the sea to the east. There is no precedent related to the application of these methodologies in the study area, therefore this study is of interest to increase our knowledge of the coastal hydrodynamics.

Keywords: Buenos Aires; coastal aquifer; electrical resistivity tomography; radon; submarine groundwater discharge.

\section{Introducción}

La interacción entre el agua subterránea y el agua de mar a lo largo de la zona costera es un fenómeno complejo que involucra muchas variables y el cual está sólo parcialmente comprendido. La descarga submarina (SGD por su sigla en inglés, submarine groundwater discharge) se define como el proceso natural del agua subterránea continental por el cual ésta se mueve hacia el mar. Es un proceso hidrológico que ocurre en la interfaz continente-océano y juega un papel importante en la dinámica costera.

Recientemente, el aumento del nivel del mar y los excesos en la extracción de agua subterránea para consumo humano y comercial han llevado a un descenso de los niveles hidráulicos por debajo del nivel del mar, con la consecuente contaminación del agua dulce por intrusión salina (Breier et al, 2005; Nyquist et al, 2005; Day-Lewis et al, 2006). En áreas costeras donde el agua subterránea es la única fuente de abastecimiento para uso humano, los procesos que controlan la interacción entre el agua dulce y el agua de mar son extremadamente importantes para asegurar la viabilidad futura de las comunidades costeras. EI Partido de La Costa (PDLC), en Buenos Aires, Argentina representa una de estas comunidades enteramente dependientes del agua subterránea para su subsistencia.

Muchas veces se realizan estudios sobre descarga desde el punto de vista de los acuíferos por medio de sus parámetros hidráulicos o desde la perspectiva marina, por ejemplo, utilizando seepage meters o trazadores químicos, pero raramente se utilizan ambos enfoques. En este trabajo se ha aplicado una combinación de técnicas, como mediciones de ${ }^{222} \mathrm{Rn}$ (trazador), resistividad eléctrica en 2D y mapas de flujo subterráneo, en la costa oriental de la provincia de Buenos Aires (Argentina) con el objetivo de detectar la descarga de agua dulce desde el acuífero arenoso hacia el Océano Atlántico. No existen antecedentes de aplicación conjunta de estas metodologías en el área, lo cual resulta en una contribución de interés para el conocimiento de la hidrodinámica costera.

\section{Área de estudio}

El área de estudio corresponde a la costa arenosa de la Provincia de Buenos Aires, Argentina (PDLC) (Figura 1). Este es uno de los destinos turísticos más importantes del país, no existen industrias, ganadería, agricultura u otras actividades económicas importantes, siendo el uso del agua casi exclusivamente para consumo humano y recreativo. La población (70.000), que depende del acuífero costero para el suministro de agua, se incrementa notablemente durante el verano, llegando a quintuplicar su valor.

El clima es templado húmedo, con una estación seca en los meses fríos (abril-septiembre) y una húmeda en los meses cálidos (octubre-marzo). La precipitación media anual oscila entre los 900 y 1000 $\mathrm{mm}$, donde el $60 \%$ se produce en los meses con mayor evapotranspiración (cálidos), por lo que la mayor recarga del agua subterránea se produce durante la estación fría (Carretero y Kruse, 2012). Se reconocen dos ambientes geomorfológicos, el cordón costero y la Ilanura deprimida. El primero se caracteriza por la presencia de arenas finas con agua subterránea de baja salinidad, principalmente del tipo $\mathrm{Ca}-\mathrm{HCO}_{3}$ y $\mathrm{Na}-$ $\mathrm{HCO}_{3}$. En el segundo predominan los materiales limosos y arcillosos, y contiene agua de elevada salinidad, tipo Na-Cl (Carretero et al., 2013b).

El acuífero principal de agua dulce está constituido por arenas de médanos superpuestas a arenas de barrera, con un espesor promedio de $10 \mathrm{~m}$, y está limitado por dos interfaces, hacia el continente agua dulce-agua salobre y hacia el mar, agua dulce-agua salada. Este acuífero es la única fuente de provisión de agua dulce para la población.

El sector central del cordón costero es la zona de recarga principal, producida a partir de los excesos de las precipitaciones, la conducción se efectúa en un corto tramo y se da la descarga en dos direcciones opuestas, una hacia el mar y la otra al oeste hacia la llanura deprimida. En términos generales, la divisoria de agua subterránea coincide con las máximas alturas topográficas siguiendo una línea imaginaria orientada en sentido norte-sur. El espesor aprovechable del acuífero se ve limitado por una zonación química y un gradiente vertical de la conductividad eléctrica, la cual se incrementa abruptamente entre los 5-7 $\mathrm{m}$ de profundidad (Carretero et al., 2016). Se reconocen procesos puntuales de salinización particularmente en la localidad de Santa Teresita (Carretero et al., 2013a; Perdomo et al., 2013a), los cuales se vinculan con una extracción intensiva del recurso que favorece el avance de un frente salino. Esto se verifica por un fuerte incremento en los valores de conductividad eléctrica en pozos próximos a la costa.

\section{Metodología}

En marzo de 2016 se llevó a cabo una campaña en el PDLC para estudiar la SGD. Se han utilizado tres métodos de estudio: piezómetros, ${ }^{222} \mathrm{Rn}$ como trazador y 


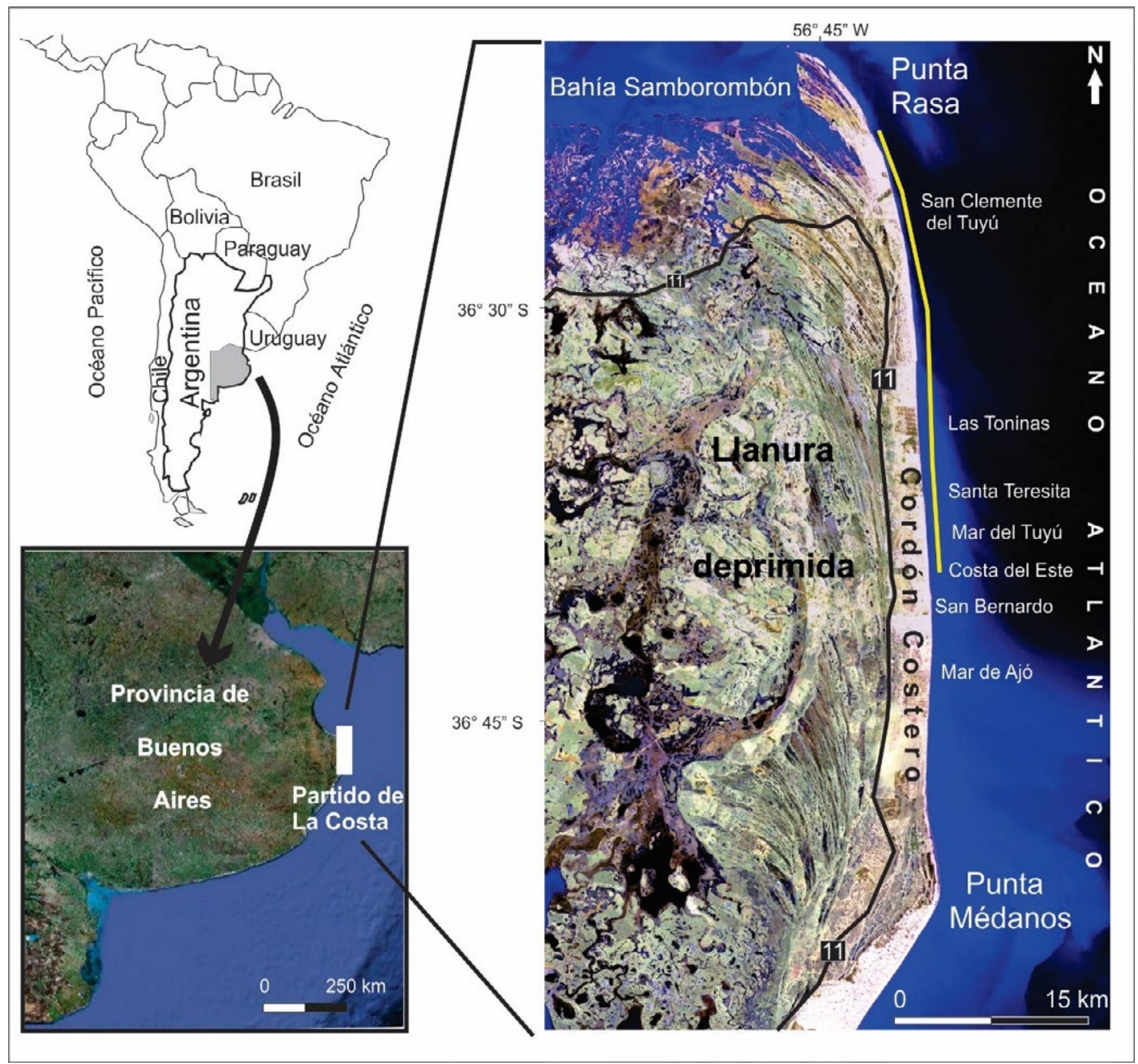

Figura 1. Área de estudio. La transecta recorrida por la embarcación está representada por la línea amarilla desde San Clemente hasta Costa del Este. Figure 1. Study area. The track followed by the boat is represented by the yellow line from San Clemente to Costa del Este.

tomografía de resistividad eléctrica. Con los dos primeros es posible realizar una cuantificación mientras que el último es cualitativo y muestra la distribución areal.

\section{Piezómetros}

Se dispone de más de 100 piezómetros distribuidos a lo largo del cordón costero, los cuales constituyen una red de monitoreo que abarca desde San Clemente hasta Mar delTuyú $\left(90 \mathrm{~km}^{2}\right)$. Este sector corresponde al $50 \%$ del área del PDLC, pero incluye más del 60 $\%$ de los sectores urbanizados. Se midieron los pozos y se confeccionaron los mapas de flujo correspondientes a cada sector a partir de los cuales se calculó la descarga hacia el mar.

Burnett et al (2006) menciona el método de los piezómetros como una de las metodologías para el cálculo de la SGD, teniendo en cuenta y considerando constante el valor de conductividad hidráulica del acuífero, utilizando la ley de Darcy de acuerdo a la ecuación: 
donde $Q$ es el flujo de Darcy (volumen de agua subterránea descargada por unidad de área por unidad de tiempo), $K$ es la conductividad hidráulica y $\mathrm{d} h / \mathrm{d} L$ es el gradiente hidráulico, en donde $h$ es la altura hidráulica y $L$ la distancia. Para el área de estudio se ha considerado una $K$ de $20 \mathrm{~m} / \mathrm{d}$.

\section{Radón}

La medición de radón en el agua de zonas costeras es una técnica para localizar potenciales fuentes de SGD (Stieglitz, 2005, Rapaglia et al., 2015). Se midió el $\mathrm{Rn}$ en forma constante utilizando un sistema de detección de $\mathrm{Rn}$ en el aire (RAD7), modificado para medir agua vía intercambio a través de un mini-módulo con filtro de membrana (Liqui-Cel). Se navegó en una embarcación de pequeño porte en forma paralela a la costa desde Punta Rasa hasta Costa del Este $(30 \mathrm{~km})$ a una velocidad promedio de 3 nudos, tratando de mantener una distancia menor a $500 \mathrm{~m}$ de la costa. Se bombeó el agua al mini-módulo utilizando una bomba peristáltica a una tasa de 0,5-0,8 $\mathrm{L} / \mathrm{min}$, devolviendo el excedente al mar. La actividad del Rn fue continuamente medida por el RAD7 según la técnica descripta por Burnett y Dulaiova (2003). Simultáneamente se midió temperatura y salinidad utilizando un conductívímetro eléctrico marcaYSI ProODO. En tres ocasiones el sistema cerrado estalló debido a la carga de sedimentos en suspensión proveniente de la descarga del Río de la Plata. En cada oportunidad el bote se detuvo hasta que el equilibrio fue alcanzado nuevamente. Desafortunadamente no se tomaron muestras para obtener la concentración de los sedimentos en suspensión. La velocidad del viento fue muy baja $(\sim 1-3$ $\mathrm{m} / \mathrm{s}$ ) y la temperatura del aire fluctuó entre $15^{\circ} \mathrm{C}$ en la mañana y $28^{\circ} \mathrm{C}$ en la tarde. La actividad del Rn fue posteriormente corregida por desgasificación debido a estos dos factores. También se midió la conductividad eléctrica in situ y la actividad del $\mathrm{Rn}$ directamente en la playa, tanto en la zona intermareal como en la zona de surf y en varias perforaciones lo largo del PDLC. El agua de las perforaciones fue bombeada a través del mini-modulo hasta que se alcanzó la concentración en equilibrio en el detector (aproximadamente 35 minutos).

\section{Geofísica: tomografía de resistividad eléctrica (ERT)}

La tomografía de resistividad eléctrica, también conocida por el acrónimo ERT por denominación en inglés (electrical resistivity tomography) resulta una herramienta eficaz y expeditiva para estudiar el subsuelo. En particular se ha utilizado exitosamente en diversos trabajos para la caracterización de la relación agua dulce-agua salada (Perdomo et al., 2013a; Perdomo et al., 2013b) y la identificación de la descarga de agua dulce (Andersen et al., 2007). Las mediciones de resistividad se integran en un perfil de 2D, por lo que permite estudiar variaciones laterales y verticales de esta propiedad en el subsuelo, que pueden ser causadas por cambios en la litología, granulometría e incluso en las características químicas del agua subterránea.

Las determinaciones de la resistividad se realizaron con un resistivímetro de corriente continua conectado a un navegador GPS para georreferenciar cada medida. Se utilizó un cable multinúcleo arrastrado desde el bote, lastrado y compuesto por cinco electrodos metálicos de acero inoxidable que estuvieran en contacto con el fondo marino. y se materializó un dispositivo electródico polo-dipolo con una distancia mínima entre electrodos de $5 \mathrm{~m}$ (Figura 2). El electrodo de infinito se ubicó cerca del bote a $40 \mathrm{~m}$ de distancia del arreglo, las distancias reales fueron consideradas para el cálculo de las constantes geométricas.

Se registraron 11607 datos de resistividad aparente a lo largo de un perfil de aproximadamente $35 \mathrm{~km}$, hasta 3 niveles de medición y un espaciado aproximado de $10 \mathrm{~m}$ entre mediciones consecutivas. Las medidas de resistividad aparente fueron procesadas con el software Res2dlnv (Loke, 2015). En primera instancia se consideraron secciones de $3 \mathrm{~km}$ de largo donde la distancia a la costa había sido constante, y también reducir los tiempos de inversión. En la etapa de procesamiento se consideró el efecto de la capa de agua de mar por encima de los electrodos de medición, incorporando la batimetría del fondo marino y la conductividad eléctrica medida in-situ. La rutina de inversión utilizada por el programa se basa en el método de mínimos cuadrados suavizados restringidos (deGroot-Hedlin y Constable, 1990; Sasaki, 1992), y básicamente el algoritmo trata de reducir la diferencia entre los valores de resistividad aparente medidos y calculados mediante el ajuste de la resistividad del modelo. La medida de esta diferencia

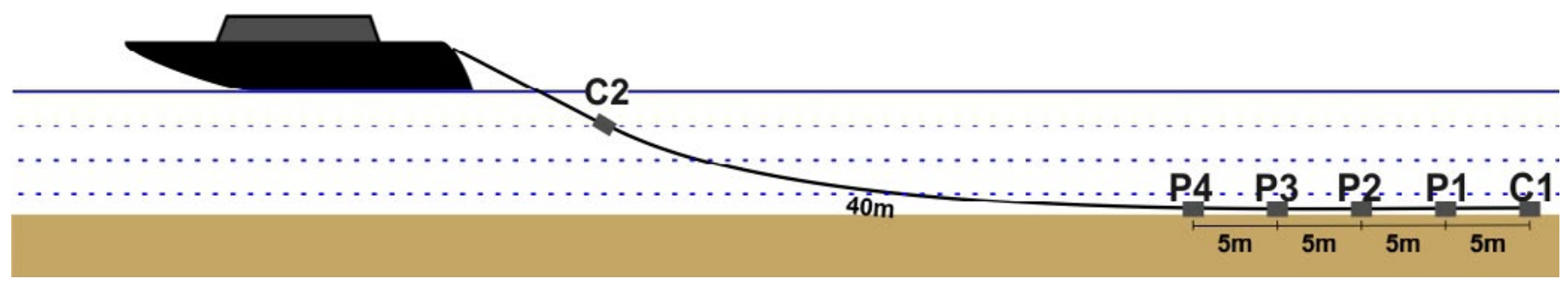

Figura 2. Disposición de los electrodos para el arreglo Polo-Dipolo. Figure 2. Arrangement of the electrodes for the pole-dipole array. 
viene dada por el error medio cuadrático (RMS) que en inversiones de datos experimentales se acepta un $5 \%$ como error máximo tolerable.

\section{Resultados}

\section{Hidrodinámica}

En los mapas de flujo confeccionados se observa que las curvas isofreáticas muestran un incremento de norte a sur. Respecto a la descarga regional, existen dos direcciones preferenciales, hacia el oeste, a la llanura deprimida, y hacia el este, al mar. El presente estudio se enfoca en esta última (Figura 3).

En el sector de San Clemente los niveles oscilan entre 0,5 y 2 m s.n.m., con las mayores alturas al sur de la localidad. Se observan dos conos de depresión relacionados a la extracción de agua del campo de bombeo (sur) y de un pozo municipal (norte). El gradiente hidráulico medio es de 0.0014 . Las Toninas se caracteriza por niveles entre 1,5 y $3 \mathrm{~m}$ s.n.m., con gradientes hidráulicos de 0,0022 . Santa Teresita presenta los niveles más bajos; incluso existen sectores de la costanera con pozos con valores por debajo del nivel medio del mar, donde existe un proceso contaminación del acuífero por intrusión salina. Las alturas máximas de la capa freática en esta localidad están representadas por las curvas de 1,5 m s.n.m. y el gradiente hidráulico resulta 0,0019. Mar del Tuyú presenta curvas entre 1,5 y $4 \mathrm{~m}$ s.n.m., resultando en la localidad con las mayores alturas de la capa freática, con un gradiente hidráulico de 0,0033.

Los valores de caudales de descarga calculados a partir de los mapas de flujo, expresados en $\mathrm{m}^{3} / \mathrm{d}$ por
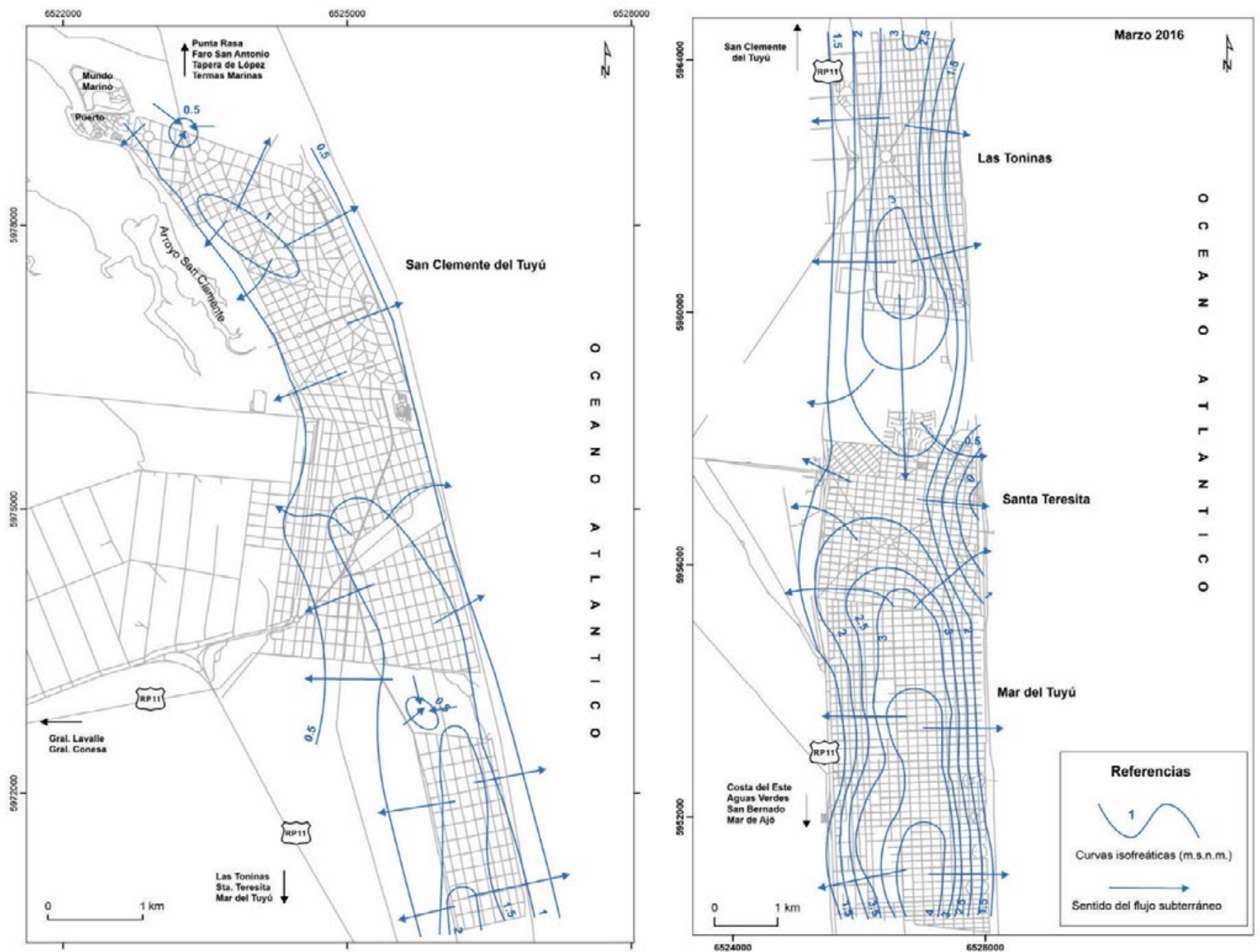

Figura 3. Mapas de flujo para marzo de 2016. A la izquierda, la localidad de San Clemente del Tuyú, a la derecha sector que abarca Las Toninas, Sta. Teresita y Mar delTuyú.

Figure 3. Goundwater flow maps for March 2016. Left: San Clemente del Tuyú town. Right: sector including the towns of Las Toninas, Sta. Teresita and Mar delTuyú. 
metro de costa $\left(\mathrm{m}^{3} / \mathrm{m} / \mathrm{d}\right)$, son 0,$21 ; 0,41 ; 0,38$ y 0,57 para San Clemente, Las Toninas, Santa Teresita y Mar delTuyú respectivamente. El caudal promedio de descarga para toda el área sería de $0,37 \mathrm{~m}^{3} / \mathrm{m} / \mathrm{d}$. La SGD anual resulta en $574 \mathrm{~m}^{3} / \mathrm{m} / \mathrm{a}$, lo que contabiliza un total de $12.400 .000 \mathrm{~m}^{3} / \mathrm{a}\left(12,4 \mathrm{hm}^{3} / \mathrm{a}\right)$ para todo el sector estudiado.

\section{Radón}

La actividad del Rn generalmente decrece con la distancia a la desembocadura del Río de la Plata (Punta Rasa). Si bien no se tomaron muestras para evaluar la cantidad de sedimentos en suspensión en las aguas, visualmente se observó una disminución en la turbidez al alejarse de la influencia del río. Este gradiente de turbidez parece correlacionarse con la actividad del $\mathrm{Rn}$. Los valores decrecen de 20,5 a $1,3 \mathrm{dpm} / \mathrm{L}$ conforme el desplazamiento a lo largo de la costa. Se detectaron pequeñas fluctuaciones en el km 12 y 20 de la transecta.

En el continente, la actividad del $\mathrm{Rn}$ en los pozos resultó ser bastante estable. Generalmente existe una alta variabilidad entre los extremos, pero en este caso resultó ser pequeña, fluctuando alrededor de un $20 \%$. Los valores promedio son de $130 \mathrm{dpm} / \mathrm{L}$, con excepción del pozo SC17, el cual presentó una baja actividad y fue dificultoso el bombeo (Rn $\sim 15 \mathrm{dpm} / \mathrm{L})$. No se ha observado ninguna tendencia en la distribución espacial de la actividad del Rn en el PDLC ni tampoco correlación con la salinidad del agua subterránea.

La actividad del $\mathrm{Rn}$ en la zona intermareal ( 50 $\mathrm{dpm} / \mathrm{L})$ y en la zona de surf $(\sim 30 \mathrm{dpm} / \mathrm{L})$ fue relativamente alta si se la compara con los valores encontrados en la transecta. Cabe aclarar que las mediciones de $\mathrm{Rn}$ en el aire fueron despreciables $(\sim 0.3 \mathrm{dpm} / \mathrm{L})$.

Resulta complejo cuantificar la SGD utilizando la actividad del $\mathrm{Rn}$ a lo largo de la costa, debido a que la mezcla de aguas es difícil de caracterizar y el componente de entrada en el balance de masas no es tan claro como sería en el caso, por ejemplo, de una laguna costera (Stieglitz et al., 2010). Sin embargo, asumiendo que el aporte de $\mathrm{Rn}$ desde mar abierto sea despreciable, se puede calcular un balance de masas para intentar una cuantificación.

Las fuentes y sumideros de ${ }^{222} \mathrm{Rn}$ en aguas costeras puede representarse por el modelo de balance de masas de Burnett and Dulaiova (2003).

$$
F_{-} S G D+F \_D i f f+F_{-} R i v=F_{-} a t m+F_{-} d e c+F \_ \text {mix }
$$

Donde $\mathrm{F}_{\mathrm{SGD}}=$ flujo de ${ }^{222} \mathrm{Rn}$ desde la SGD $\left(\mathrm{dpm} / \mathrm{m}^{2} / \mathrm{d}\right)$, $\mathrm{F}_{\text {diff }}=$ flujo de ${ }^{222} \mathrm{Rn}$ por difusión desde los sedimentos, $\mathrm{F}_{\mathrm{Riv}}={ }^{222} \mathrm{Rn}$ aportado desde el río; $\mathrm{F}_{\mathrm{atm}}=$ pérdida de ${ }^{222} \mathrm{Rn}$ por difusión atmosférica; $F_{\text {dec }}=$ desintegración del ${ }^{222} \mathrm{Rn}$ en la columna de agua; $F_{\text {mix }}=$ pérdida de ${ }^{222} \mathrm{Rn}$ debido a la mezcla.

Se asume que $F_{\text {diff }}$ no presenta una importancia sig- nificativa de acuerdo a las diferencias regionales en la distribución del ${ }^{222} \mathrm{Rn}$ que se observan en este trabajo. El aporte desde el río debería ser también despreciable, sin embargo, debido a la carga de sedimentos en suspensión no sería el caso. El problema es que este factor no es posible de cuantificar por falta de datos, por lo tanto, no se considerará en la ecuación. De todas maneras, hay que tener en cuenta que este factor sobreestima el rol de la SGD en el área. La mezcla es otro factor que resulta dificultoso de cuantificar en una costa abierta y también se desprecia. Como una primera aproximación de los cálculos, la ecuación 2 se simplifica en:

$$
F_{-}(S G D)=F_{-} a t m+F_{-} \operatorname{dec}(E c .3)
$$

La difusión atmosférica se calcula multiplicando el coeficiente de transferencia del gas por la concentración promedio de radón en aguas abiertas. Este valor sería insignificante ya que depende de la velocidad del viento, la cual fue muy baja durante el muestreo. Por lo tanto, el mayor factor que determina el balance de masas es el decaimiento, el cual depende de la profundidad del agua y la concentración de radón en la superficie. El flujo de radón por SGD puede ser dividido por la actividad del radón en los pozos para determinar el flujo en $\mathrm{m}^{3} / \mathrm{m} / \mathrm{d}$.

Usando el promedio de la actividad del $\mathrm{Rn}$ en la transecta $(7.3 \mathrm{dpm} / \mathrm{L})$ y el promedio de los pozos (130 $\mathrm{dpm} / \mathrm{L}$ ) como valor extremo, se ha intentado calcular el flujo de descarga. Los valores indican que la SDG en el PDLC es de $1,1 \mathrm{~m}^{3} / \mathrm{s}$ o $3,5( \pm 2,5) \mathrm{m}^{3} / \mathrm{m} / \mathrm{d}$. Esto resulta en un orden de magnitud mayor que el calculado por medio de la Ley de Darcy. Esta diferencia podría atribuirse a la incertidumbre en el tiempo de residencia, en los valores extremos de radón en los pozos, la recirculación de agua de mar y complicaciones debidas a la alta turbidez del agua en el extremo norte del PDLC.

\section{Tomografía de resistividad eléctrica}

El modelo de resistividad obtenido (Figura 4) presenta una primera capa de resistividad constante de $0,25 \mathrm{Ohm} \cdot \mathrm{m}$, que corresponde al agua de mar, con una parte superior plana y la base constituida por la batimetría del fondo. Por debajo del fondo marino se observan valores entre 0,25 y $10 \mathrm{Ohm} \cdot \mathrm{m}$, para los cuales, asumiendo una uniformidad litológica de los sedimentos marinos, los valores más bajos corresponderían a la presencia de agua salada, mientras que las zonas de mayor resistividad pueden interpretarse como zonas de mezcla entre el agua salada subterránea y agua dulce producto de la descarga. A lo largo de los $35 \mathrm{~km}$ se observan estas anomalías más resistivas entre los 3 y $4 \mathrm{~m}$ de profundidad por debajo del fondo marino y se encuentran ausentes entre las distancias 16320 y 20160 m, próximo a la localidad de LasToninas y hacia el final del perfil en la localidad de Costa del Este, donde la hidrodinámica y el radón sí 
indican la existencia de SGD. Este fenómeno posiblemente se deba a que en dicho tramo la velocidad de navegación fue levemente mayor que en el resto del perfil. Esto podría haber elevado los electrodos de medición que iban arrastrados y en contacto con los sedimentos y así evitar que la corriente circule por el subsuelo y dificultar la detección de la zona de mayor resistividad.

Se observan sectores con anomalías de valores de resistividad superior a los $5 \mathrm{Ohm}$.m, en las distancias $3520,9920,15040$ y $24000 \mathrm{~m}$, que podrían estar asociadas a un aumento en el espesor o mayor proporción de agua dulce en la zona de mezcla.

\section{Discusión}

Mediante la utilización de los tres métodos planteados se ha detectado la SGD a lo largo de la costa bonaerense. En una primera instancia se realizaron estimaciones cuantitativas con dos metodologías que han dado resultados con diferencias en un orden de magnitud. Las mediciones de niveles freáticos y el cálculo por medio de la ecuación de Darcy se vienen utilizando en el área de estudio desde hace más de 10 años. Los valores obtenidos presentan una variabilidad temporal que depende de los ciclos hidrológicos, pero se mantienen siempre dentro de rangos del mismo orden por lo cual esta metodología se considera consistente.

La aplicación del ${ }^{222} \mathrm{Rn}$ para el cálculo de la SGD ha dado resultados que difieren de la otra metodología. Si bien esta técnica es ampliamente utilizada, los me- jores resultados se obtienen en ambientes más restringidos como las lagunas costeras. En el caso particular del área de estudio, la mayor incertidumbre es el aporte de ${ }^{222} \mathrm{Rn}$ desde los sedimentos en suspensión del Río de la Plata. Es de destacar que cuanto más cerca de la desembocadura (Bahía de Samborombón) se realizó la medición, más altos resultaron los valores $\mathrm{de}^{222} \mathrm{Rn}$ los cuales fueron descendiendo a medida que la embarcación se fue alejando del frente de turbidez (Figura 5). Se plantea como tarea a futuro, para continuar con la línea de investigación, realizar muestreos para calcular la cantidad de sedimento en suspensión y ajustar este factor. También se propone continuar con la transecta hacia el sur donde al parecer ya no se detectaría la influencia de estos sedimentos, y corroborar si los valores de descarga son coincidentes por ambos métodos.

Para realizar estimaciones con la ERT es necesario conocer parámetros petrofísicos o características granulométricas y de empaquetamiento de los sedimentos. Por tratarse de sedimentos de fondo marino, resulta compleja y costosa su determinación. Sin embargo, los modelos de la ERT han resultado ser un excelente complemento en la detección de la SGD. En la Figura 5 puede observarse la correlación entre las líneas de flujo subterráneo y los sectores con resistividades entre 0,25 y $10 \mathrm{Ohm} \cdot \mathrm{m}$ que se asume zona de descarga de agua dulce al mar.

Los mayores valores de resistividad a la distancia de 3520 m incluyen a un campo de dunas al norte de San Clemente, así como al sector a $15040 \mathrm{~m}$ al sur de

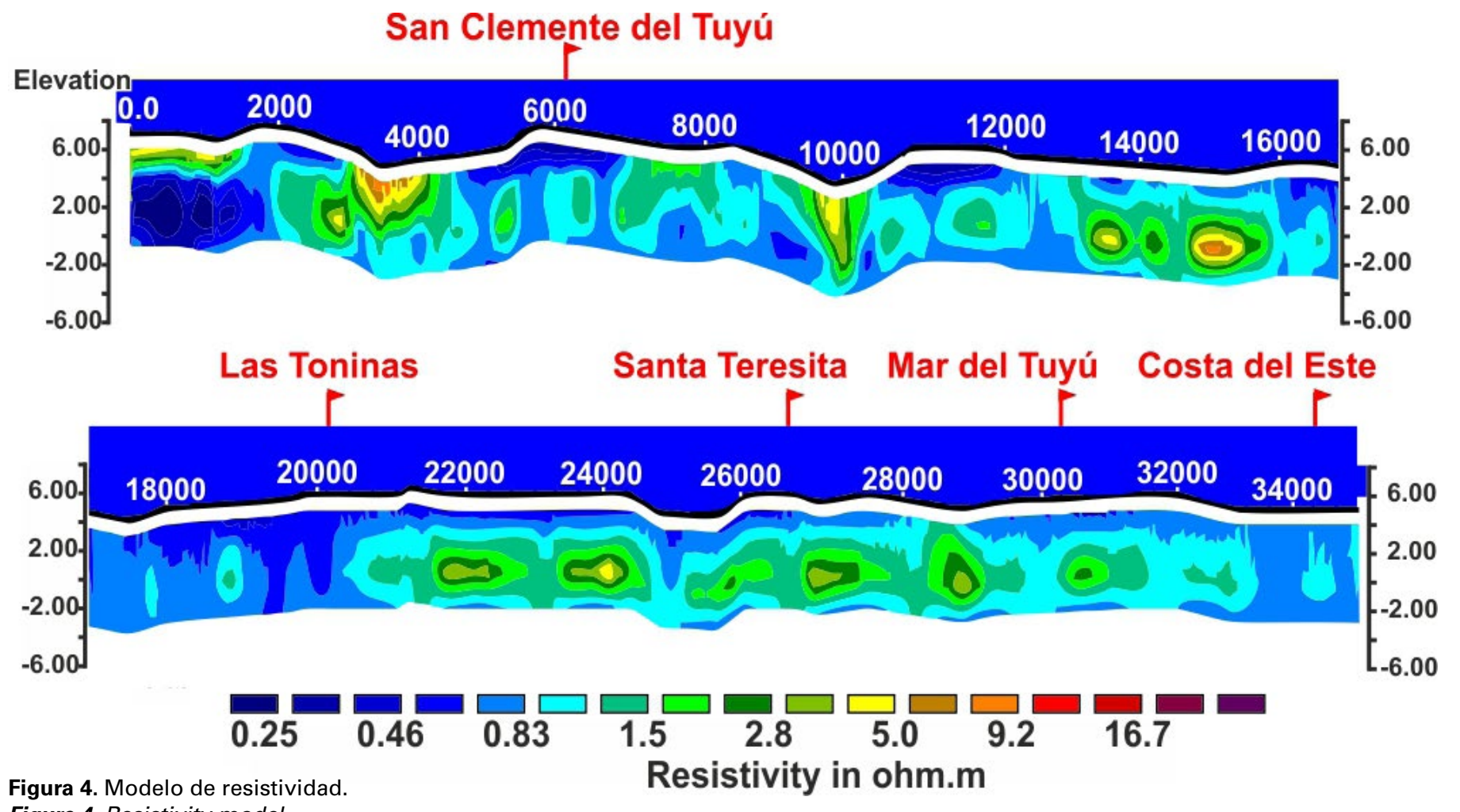




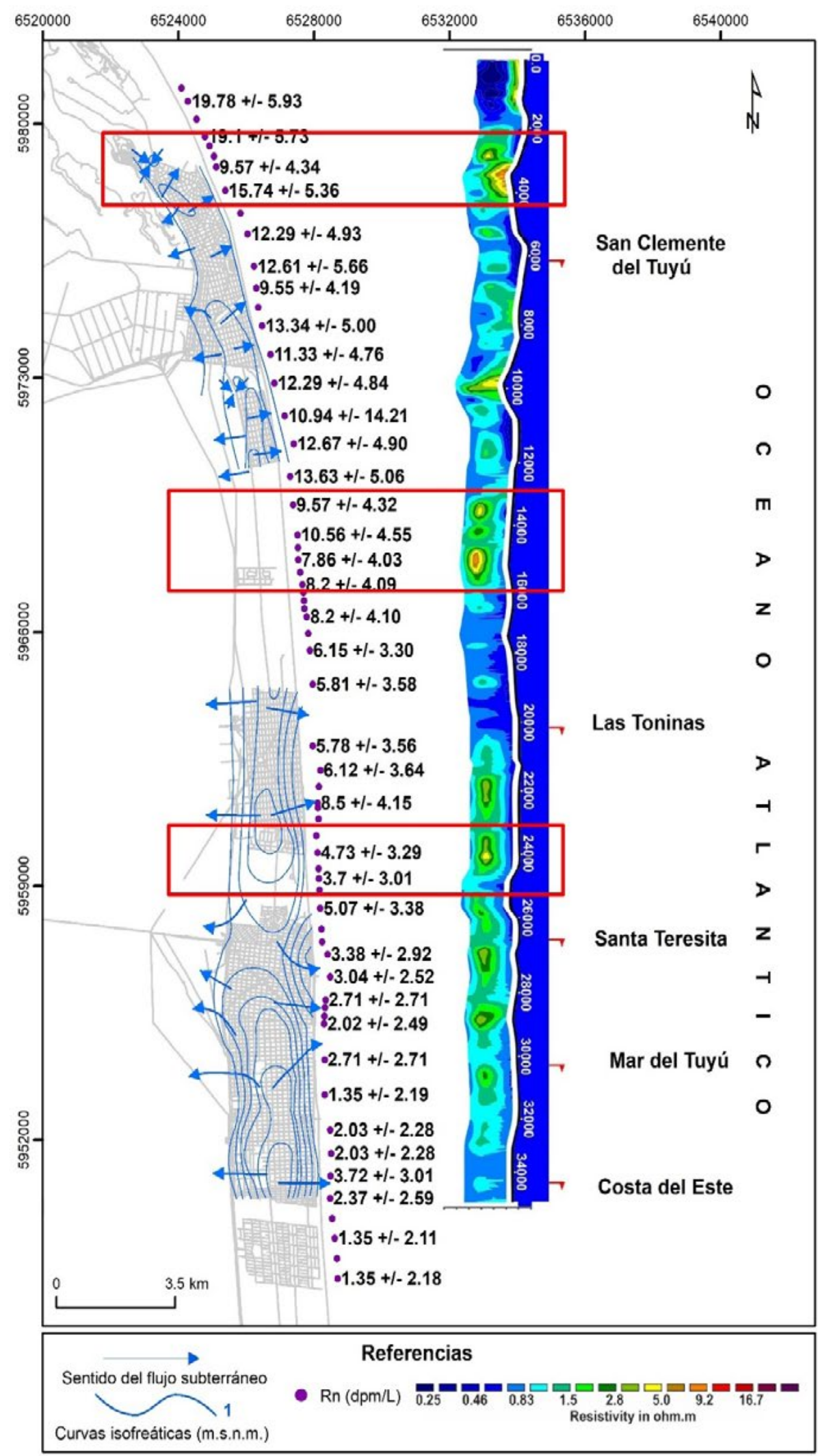

Figura 5. Mapa que indica la correlación entre las líneas de flujo del agua subterránea, la actividad del ${ }^{222}$ Rn y las zonas preferentes de SGD detectadas a partir de la ERT.

Figure 5. Map showing the correlation between groundwater flow lines, ${ }^{222}$ Rn activity and preferential SGD zones detected from the ERT. 
la misma localidad donde no hay urbanización y por lo tanto las reservas de agua serían superiores, con mayores gradientes hidráulicos y consecuentemente mayor descarga. El mismo caso se da a los $24000 \mathrm{~m}$ asociado a la zona entre Las Toninas y Santa Teresita también sin urbanización y extracción de agua subterránea.

Existe una zona frente a Santa Teresita donde es reconocido el fenómeno de intrusión marina con una extensión lateral de $900 \mathrm{~m}$ aproximadamente. En la ERT no se observó una anomalía que pudiera atribuirse a esta situación. Una posible explicación es que el fenómeno no se detecte desde la distancia a la costa en la cual se realizó la transecta.

\section{Conclusiones}

La SGD en el área de estudio ha sido confirmada mediante tres enfoques complementarios. La hidrodinámica y el uso de radón permitieron realizar una cuantificación, mientras que el método geofísico resulta más cualitativo y muestra la distribución areal de la SGD a lo largo de la línea de costa.

Los modelos 2D obtenidos a partir de la inversión de las medidas de resistividad eléctrica aparente permitieron identificar una zona por debajo del fondo marino con resistividad más alta que la del agua de mar, que podría atribuirse a una zona de mezcla debido al aporte de agua dulce. Esta zona se encuentra presente a lo largo de prácticamente todo el perfil entre los 3 y $4 \mathrm{~m}$ de profundidad debajo de la superficie del fondo.

La cuantificación ha dado valores de $0,37 \mathrm{~m}^{3} / \mathrm{m} / \mathrm{d}$ según el flujo de Darcy y de $1,1 \mathrm{~m}^{3} / \mathrm{s}$ o $3,5( \pm 2,5) \mathrm{m}^{3} / \mathrm{m} / \mathrm{d}$ con el uso del Rn, lo cual es un orden de magnitud mayor. Se plantea continuar con los estudios y testeos de las variables para disminuir estas incertidumbres a futuro.

\section{Referencias}

Andersen, M.S.; Baron, L.; Gudbjerg, J.; Gregersen, J.; Chapellier D. and Jakobsen, R. 2007. Discharge of nitrate-containing groundwater into a coastal marine environment. Journal of Hydrology (336): 98-114.

Breier J.A.; Breier C.F. and Edmond, H.N. 2005. Detecting submarine groundwater discharge with synoptic surveys of sediment resistivity, radium, and salinity. Geophysical Research Letters, 32 (23): 1-4.

Burnett, W.C. and Dulaiova, H., 2003. Estimating the dynamics of groundwater input into the coastal zone via continuous radon-222 measurements. Journal of Environmental Radioactivity 69(1-2): 21-35.

Burnett;W. C; Aggarwal. P. K.; Kulkarni K. M.; Aureli A.; Bokuniewicz H.; Cable J. E.; Charette M. A.; Kontar E.; Krupa S.; Loveless A.; Moore W. S.; Oberdorfer J. A.; Oliveira J.; Ozyurt N.; Povinec P.; Scholten J.; Privitera A. M.G.; Rajar R.; Ramessur R. T.; Stieglitz T.; Taniguchi M.and Turner P.V. 2006. Quantifying submarine groundwater discharge in the coastal zone via multiple methods. Science of the Total Environment: 67 (2-3): 498-543.

Carretero, S. and Kruse, E. 2012. Relationship between precipitation and water-table fluctuation in a coastal dune aquifer: northeastern coast of the Buenos Aires province, Argentina. Hydrogeology Journal (20): 1613-1621.

Carretero, S.; Kruse E. and Rojo, A. 2013a. Condiciones hidrogeológicas en Las Toninas y Santa Teresita, Partido de La Costa. In: N. González, E. Kruse, M.M. Trovatto y P. Laurencena (Editores) Temas actuales en hidrología subterránea 2013. ISBN 978987-1985-03-6. La Plata, EDULP, pp 28-35.

Carretero, S.; Dapeña, C. and Kruse, E. 2013b. Hydrogeochemical and isotopic characterisation of groundwater in a sand-dune phreatic aquifer in the northeastern coast of the province of Buenos Aires. Argentina. Isotopes in Environmental \& Health Studies 49(3): 399-419.

Carretero, S.; Perdomo S.; Kruse, E. and Ainchil, J. 2016. Respuesta eléctrica de la zonación química en un nivel acuífero en la costa arenosa oriental de la Provincia de Buenos Aires. In: García R. y Mariño E. (Editores) Calidad del agua subterránea. Editorial Científica Universitaria - Secretaría de Ciencia y Tecnología Universidad Nacional de Catamarca, pp 93-100. San Fernando del Valle de Catamarca, Catamarca.

Day-Lewis, F.D.;White, E.A.; Johnson, C.D.; Lane, J.W. and Belaval, M. 2006 Continuous resistivity profiling to delineate submarine groundwater discharge-Examples and limitations. Leading Edge, 25 (6): 673-792.

deGroot-Hedlin, C. and Constable, S. 1990. Occam's inversion to generate smooth, two dimensional models form magnetotelluric data. Geophysics (55): 1613-1624.

Loke, M.H. 2015. Tutorial: 2-D and 3-D electrical imaging surveys. Geotomo Software, Malaysia.

Nyquist, J.E.; Freyer, P.A. and Toran, L. 2005. Stream Bottom Resistivity Tomography to Map Ground Water Discharge, Groundwater, 46 (4): 561-569.

Perdomo, S.; Carretero, S.; Kruse, E. and Ainchil, J. 2013 a. Identificación de la intrusión salina en Santa Teresita (Buenos Aires), mediante la aplicación de métodos eléctricos. In: N. González, E. Kruse, M.M. Trovatto y P. Laurencena (Editores) Temas actuales en hidrología subterránea 2013. EDULP, pp 44-49. La Plata.

Perdomo S., Rodrigues Capítulo L., Kruse E. and Ainchil J., 2013b. Aplicación de tomografías eléctricas en la configuración del acuífero costero en un sector oriental de la Provincia de Buenos Aires. In: N. González, E. Kruse, M.M. Trovatto y P. Laurencena (Editores) Temas actuales en hidrología subterránea 2013. EDULP, pp 57 - 62. La Plata.

Rapaglia, J., Grant, C., Bokuniewicz, H., Pick, T. and Scholten, J. 2015. A GIS typology to locate sites of submarine groundwater discharge. Journal of Environmental Radioactivity, 145(0): 10-18.

Sasaki, Y. 1992. Resolution of resistivity tomography 
inferred from numerical simulation. Geophysical Prospecting, 40: 453-464.

Stieglitz, T. 2005. Submarine groundwater discharge into the near-shore zone of the Great Barrier Reef, Australia. Marine Pollution Bulletin, 51(1-4): 51.
Stieglitz, T.C.; Cook, P.G. and Burnett, W.C., 2010. Inferring coastal processes from regional-scale mapping of 222Radon and salinity: examples from the Great Barrier Reef, Australia. Journal of Environmental Radioactivity, 101(7): 544-552.

Recibido: julio 2019

Revisado: noviembre 2019

Aceptado: enero 2020

Publicado: marzo 2021 